\title{
Prognostic factors predicting an unprovoked seizure recurrence in children and adults following a first unprovoked seizure (Protocol)
}

Adan G, Neligan A, Nevitt SJ, Pullen A, Sander JW, Marson AG

Adan G, Neligan A, Nevitt SJ, Pullen A, Sander JW, Marson AG.

Prognostic factors predicting an unprovoked seizure recurrence in children and adults following a first unprovoked seizure (Protocol).

Cochrane Database of Systematic Reviews 2021, Issue 1. Art. No.: CD013848.

DOI: 10.1002/14651858.CD013848.

www.cochranelibrary.com

Prognostic factors predicting an unprovoked seizure recurrence in children and adults following a first unprovoked seizure (Protocol) 
TABLE OF CONTENTS

HEADER 1

ABSTRACT

BACKGROUND

OBJECTIVES

METHODS

ACKNOWLEDGEMENTS

REFERENCES

APPENDICES

HISTORY

CONTRIBUTIONS OF AUTHORS

DECLARATIONS OF INTEREST

SOURCES OF SUPPORT 
[Prognosis Protocol]

\section{Prognostic factors predicting an unprovoked seizure recurrence in children and adults following a first unprovoked seizure}

Guleed Adan1,2, Aidan Neligan³,4, Sarah J Nevitt5, Angie Pullen6, Josemir W Sander4,7, Anthony G Marson 1,2,8

1Department of Molecular and Clinical Pharmacology, Institute of Translational Medicine, University of Liverpool, Liverpool, UK. 2The Walton Centre NHS Foundation Trust, Liverpool, UK. ${ }^{3}$ Homerton University Hospital, NHS Foundation Trust, London, UK. ${ }^{4}$ Department of Clinical and Experimental Epilepsy, UCL Queen Square Institute of Neurology, London, UK. 5Department of Health Data Science, University of Liverpool, Liverpool, UK. ${ }^{6}$ Epilepsy Action, Leeds, UK. ${ }^{7}$ National Hospital for Neurology and Neurosurgery, London, UK. 8Liverpool Health Partners, Liverpool, UK

Contact address: Aidan Neligan, a.neligan@ucl.ac.uk.

Editorial group: Cochrane Epilepsy Group.

Publication status and date: New, published in Issue 1, 2021.

Citation: Adan G, Neligan A, Nevitt SJ, Pullen A, Sander JW, Marson AG. Prognostic factors predicting an unprovoked seizure recurrence in children and adults following a first unprovoked seizure (Protocol). Cochrane Database of Systematic Reviews 2021, Issue 1. Art. No.: CD013848. DOI: 10.1002/14651858.CD013848.

Copyright $(2021$ The Cochrane Collaboration. Published by John Wiley \& Sons, Ltd.

\section{A B S T R A C T}

\section{Objectives}

This is a protocol for a Cochrane Review (prognosis). The objectives are as follows:

\section{Primary objectives}

To identify which prognostic factors predict whether individuals will go on to have further unprovoked seizures and the development of epilepsy at any subsequent time point following a single unprovoked seizure (or cluster of epileptic seizures within a 24-hour period, or a first episode of status epilepticus), of any seizure type.

\section{Investigation of sources of heterogeneity between studies}

We anticipate that there will be heterogeneity between studies, particularly in studies that have focused on adults compared to the paediatric population, and studies that have a combination of paediatric and adult populations. 


\section{B A C K G R O U N D}

Epilepsy, clinically defined after two or more unprovoked epileptic seizures, is one of the most common neurological disorders worldwide, with significant psychosocial sequelae; it has an estimated incidence of 50 to 70 per 100,000 person years, and prevalence of 5 to 10 per 1000 persons. It affects more than 50 million people world-wide (Neligan 2012; Ngugi 2011). Given that a diagnosis of epilepsy can be associated with significant morbidity and mortality (Loiseau 1999), it is imperative that clinicians (and people with seizures and their relatives) have access to accurate and reliable prognostic estimates and models, to guide clinical practice on the risks of developing further unprovoked seizures (and by definition, a diagnosis of epilepsy) following a single unprovoked seizure.

\section{Description of the health condition and context}

The condition under study is the occurrence of a single unprovoked epileptic seizure of any semiology, and the subsequent risk of seizure recurrence of any type, within a two-year period. Seizure semiology is defined according to the recent International League Against Epilepsy (ILAE) classification of seizures (Scheffer 2017). Epileptic seizures are synchronous and excessive discharges in the cerebral cortex leading to a clinically discernable event. There are many seizure types, depending on the area of the cerebral cortex in which the discharges originate. Seizures can be broadly subclassified into focal onset or generalised seizures, depending on whether the epileptic focus originates in a localised area in one cerebral cortex, as in focal onset seizures, or from both hemispheres simultaneously, as in generalised seizures. Focal seizures can be subdivided into seizures with and without impaired consciousness, depending on how localised and widespread the epileptic focus is. Seizures may take the form of short sensory, motor, or psychic symptoms, typically lasting 15 to 30 seconds, which resolve without cognitive sequelae, or progress to an episode of impaired, or complete loss of consciousness. All focal onset seizures have the potential to evolve from a state without impaired consciousness, to one with impaired consciousness, or complete loss of consciousness (focal to bilateral tonic clonic seizure), as a result of the localised epileptic focus spreading to a more widespread area, or to the opposite cerebral hemisphere.

Focal seizures with impaired consciousness, which predominantly arise from the temporal or frontal lobes, are said to occur when the person is less responsive, or more commonly, completely unresponsive to external stimuli, with or without prominent motor symptoms. These seizures can be short (15 to 30 seconds in frontal seizures, often with hypermotor activity), or more prolonged (two to four minutes with temporal seizures, often with oral or manual symptoms), following which there may be a period of confusion lasting several minutes, and amnesia for the episode. Generalised seizures, which can occur without warning, or evolve from a more focal seizure (focal to bilateral tonic clonic seizures typically involve loss of tone (atonia) and posture, with bilateral convulsive movements (tonic clonic movements) lasting several minutes, during which there may or may not be associated tonguebiting, or incontinence (urinary, or faecal, or both), or both. A typical generalised seizure lasts up to five minutes, following which there is a prolonged period of drowsiness and confusion, which lasts from minutes to hours, during which the person may sleep. People may have a headache or generalised muscle aching following a generalised seizure. Generalised seizures may have isolated features of a generalised tonic clonic seizure, such as atonia (atonic seizures), a tonic phase (tonic seizures), or a clonic phase (clonic seizures). Other generalised seizure types include absence seizures (brief staring episodes without a significant component, lasting less than a minute, often occurring in children), and myoclonus (brief involuntary contraction of a single muscle, or group of muscles).

\section{Description of the prognostic factors}

The following prognostic factors (PF) are of interest to this review:

- age of seizure onset (childhood (1 month to 16 years), or adult (> 16 years))

- gender

- seizure semiology

- electroencephalogram (EEG) findings, and in particular epileptic syndromes, such as the childhood epileptic encephalopathies

- clinically relevant abnormal findings on magnetic resonance imaging (MRI), including specific aetiologies, such as hippocampal sclerosis, cortical dysplasia, dysembryoplastic neuroepithelial tumours (DNET), and cavernomas

- presence of comorbidities, such as learning disability

- specific subpopulations, or people with specific aetiologies, such as traumatic brain injury, and people with established cerebrovascular disease, or neuro-generative conditions

- impact of treatment, such as anti-seizure drugs (ASMs), initiated following a single seizure

- specific pre-identified genetic abnormalities (for example the KRT1 gene in people with multiple cavernomas, or children with learning disabilities and pre-identified genetic mutations, such as CDKL5 deficiency disorder or PCHD19 epilepsy)

\section{Health outcomes}

The risk factors listed above are believed to potentially influence the risk of subsequent, unprovoked seizures of any type, and have been examined in previous studies, such as the Medical Research Council's Multicentre trial for early epilepsy and single seizures (MESS) study (Marson 2005). In particular, it has been shown that an abnormal EEG, abnormal neuro-imaging, or both, is associated with an increased risk of further seizures, yet it is less clear what the impact of specific aetiologies on neuro-imaging, or indeed, the risk of unprovoked seizure recurrence, is in the context of specific EEG patterns, or other risk factors.

\section{Why it is important to do this review}

It is estimated that the cumulative incidence of a single unprovoked epileptic seizure in the general population is approximately $3 \%$ to $4 \%$, by the time one reaches 85 years of age (Hauser 1993). Consequently, almost one in 25 people will have an epileptic seizure during their lifetime, and it is imperative that accurate prognostic data are available for clinicians, so they can reliably counsel people on the risk of further seizures, and factors that predict the recurrence of seizures and the development of epilepsy. People who present with a single unprovoked seizure will be typically investigated with an MRI scan, and possibly an EEG (depending on age), which is justified on prognostic grounds. Nevertheless, it is unclear what additional risk an abnormal EEG or a specific abnormality on MRI confers. If the risk is sufficiently increased, this may justify commencing antiepileptic medication after a single seizure (rather than after two or more unprovoked

Prognostic factors predicting an unprovoked seizure recurrence in children and adults following a first unprovoked seizure (Protocol) 
seizures more than 24 hours apart, as is standard practise). People presenting with a single seizure, their families, and the clinicians looking after them, deserve more accurate prognostic estimates of the risk factors associated with further, unprovoked seizures, and the development of epilepsy.

This review will focus on individual prognostic factors, in isolation and in combination, that influence the risk of seizure recurrence following an single unprovoked epileptic seizure.

\section{O B JECTIVES}

\section{Primary objectives}

To identify which prognostic factors predict whether individuals will go on to have further unprovoked seizures and the development of epilepsy at any subsequent time point following a single unprovoked seizure (or cluster of epileptic seizures within a 24-hour period, or a first episode of status epilepticus), of any seizure type.

\section{Investigation of sources of heterogeneity between studies}

We anticipate that there will be heterogeneity between studies, particularly in studies that have focused on adults compared to the paediatric population, and studies that have a combination of paediatric and adult populations.

\section{METHODS}

This review will be conducted within the framework of the Cochrane Review Group, and reported in line with the PRISMA guidelines (Moher 2009). The 'Methods' section is based on the exemplar Cochrane Prognosis Review protocol for prognostic factors (Hayden 2014), and the general protocol template of the Cochrane Prognosis Methods Group.

\section{Criteria for considering studies for this review}

Population: children ( 1 month to 16 years) and adults (> 16 years) with a previous unprovoked epileptic seizure of any semiology. It is anticipated that the studies we include will examine either exclusively paediatric or adult cohorts, as is the norm in epilepsy studies.

Intervention: this is a review of observational studies, with no active intervention.

Comparator: the comparison will be an internal group comparison between those with a seizure recurrence compared to those without.

Outcome: the primary outcome is recurrence of a further unprovoked seizure of any semiology, and the identification of prognostic factors that predict such an outcome.

Timing: any seizure recurrence of any semiology, more than 24 hours from the index seizure, in studies with a minimum of six months of follow-up, with no upper time limit for inclusion

Settings: hospital outpatient or community settings

\section{Types of studies}

We will include all cohort studies, both retrospective and prospective, of all age groups, excluding those in the neonatal period ( $<1$ month of age), of people with a single unprovoked seizure (of any semiology), followed up for a minimum of six months, with no upper limit of follow-up, with the study end point being (an unprovoked) seizure recurrence, death, or loss to followup. To be included, studies must include at least 30 participants (West 2019). We will also consider well conducted case-control studies for the secondary objective of the review, where prognostic factors are well defined (see 'Assessment of risk of bias in included studies', Appendix 1 ).

\section{Targeted population}

Population and hospital cohorts of people over one month of age, presenting with a single unprovoked seizure of any semiology, with a follow-up period of at least six months.

We will exclude people wit seizures that occur as a result of an acute precipitant or provoking factor, or in close temporal proximity to an acute neurological insult (such as a head injury, acute cerebrovascular accident), since they are not considered epileptic in aetiology (acute symptomatic seizures; (Kwan 2010)). Similarly, we will exclude people with situational seizures, such as febrile convulsions, which occur in young children in the context of a high temperature.

\section{Types of prognostic or predictive factor(s) or model(s)}

We will consider the following prognostic factors for prediction of seizure recurrence: age of seizure onset, gender, seizure semiology, EEG findings, and in particular, epileptic syndromes, such as the childhood epileptic encephalopathies; clinically relevant abnormal findings on MRI, including specific aetiologies, such as hippocampal sclerosis, cortical dysplasia, dysembryoplastic neuroepithelial tumours (DNET), and cavernomas; presence of comorbidities, such as learning disabilities; specific subpopulations or people with specific aetiologies, such as those with traumatic brain injury, and people with established cerebrovascular disease or neuro-generative conditions; impact of treatment, such as anti-seizure medications (ASMs) initiated following a single seizure; specific genetic abnormalities.

\section{Types of outcomes to be predicted}

The primary outcome will be the occurrence of a second (unprovoked) epileptic seizure, more than 24 hours after the original seizure of any type.

We will analyse this as the proportion of people who have a further seizure, in any time period, with an evaluation of the impact of the individual predictive factors on this outcome; we will conduct a time-to-event analysis, if possible.

\section{Search methods for identification of studies}

\section{Electronic searches}

We will search the following databases, with no language restrictions.

1. The Cochrane Register of Studies (CRS Web), using the strategy outlined in Appendix 2; 
2. MEDLINE Ovid (1946 to search date), using the strategy outlined in Appendix 3;

3. SCOPUS (1823 to search date), using the subject and citation strategies outlined in Appendix 4;

4. ClinicalTrials.gov, using the strategy outlined in Appendix 5;

5. The World Health Organisation (WHO) International Clinical Trials Registry Platform (ICTRP), using the strategy outlined in Appendix 6.

To avoid unnecessary duplication of work, we will use the same search for both this review and the overall prognosis review (Neligan 2021). However, we anticipate several of the papers included in Neligan 2021 will not be eligible for this review.

\section{Searching other resources}

We will search for additional relevant studies in the reference lists of included studies and any relevant systematic reviews identified in the search.

\section{Data collection}

\section{Selection of studies}

A single review author (AN or GA), will conduct the initial screening of titles and abstracts identified through the electronic searches, and remove clearly irrelevant articles. We will obtain the fulltext articles of all potentially relevant studies, or those whose relevance cannot be determined from the abstract, and two authors (AN, GA) will independently assess for eligibility. They will resolve disagreements through discussion, or if required, consultation with a third review author (AGM).

When studies were reported in multiple publications or reports, we will collate all relevant reports under a single study, so that the study, rather than the report, is the unit of interest in the review.

We will outline the study selection process in a PRISMA study flow diagram (Moher 2009).

\section{Data extraction and management}

We will extract data from included studies using a data extraction form.

We will base the data extraction form on the checklist for critical appraisal and data extraction for systematic reviews of prediction modelling studies (CHARMS); we will pilot it on several studies, and make appropriate edits (Moons 2014). Two review authors (AN, GA) will extract data; a third review author (SJN) will check the data. We will resolve disagreements through discussion, or if required, by consulting with a fourth review author (AGM).

List of data to extract:

- date of first seizure and any subsequent seizures

- age

- gender

- seizure semiology - focal onset, generalised, impairment of consciousness

- result of EEG - specific findings

- result of neuro-imaging - specific findings

- proposed aetiology

- ASM therapy initiated or not
- Pre-identified specific genetic abnormalities.

We will contact trial authors for missing data. We will give them 30 days to respond, after which time, we will only include published data for the purposes of this review.

We will extract all unadjusted and adjusted measures of association from included studies, and convert effect sizes, as necessary, to avoid possible selection bias and allow us to use data from as many studies as possible.

\section{Assessment of risk of bias in included studies}

Two review authors (AN and GA) will independently appraise the included studies, using a standardised approach, based on the quality in prognostic studies (QUIPS) tool, appropriate for the prognostic factors considered in this review (Hayden 2013; Appendix 1). In the case of discrepancies, the review authors will attempt to reach consensus; where necessary, a third review author (AGM) will resolve any disagreements.

Our approach will assess the risk of bias of all prognostic studies (in addition to any missing or unclear information) for six domains of bias; study participation (selection bias), study attrition, prognostic factor measurement, outcome measurement, adjustment and statistical analysis, and reporting. We will judge each domain of bias at high, moderate, or low risk of bias, using the QUIPS tool. We will note methodological comments, including quotes from the study publications, to support our judgements.

We will also judge overall risk of bias, by defining studies with a low risk of bias as those in which we rated all six bias domains at low risk of bias. We will conduct a sensitivity analysis in which we only include studies judged to be at low risk of bias overall (Hayden 2013).

\section{Measures of association or predictive performance measures to be extracted}

We will use the odds ratio (OR) as the common measure of association. We will extract ORs, risk ratios (RRs), and hazard ratios (HRs) from univariate or multivariable regression analyses, if reported, and convert RRs and HRs to estimate ORs at a particular time point (Symons 2002). Alternatively, if prognostic factors are reported as dichotomous variables (frequencies), we will extract data for the outcomes and prognostic factors in the format of $2 \times 2$ tables, and convert to effect sizes.

For consistency, we will re-calculate associations so they are in the same direction.

When data are available, we will synthesise adequately adjusted (multivariable) associations between prognostic factors and outcomes separately from unadjusted (univariable) associations.

\section{Dealing with missing data}

We will include studies that investigate the relationship between the prognostic factors and the outcomes, even if there are missing data, or limited evidence is provided about the size of the effect (for example if the factor is mentioned only as being 'non-significant' in the analyses).

If required, we will calculate or estimate effect sizes from any data reported (e.g. $2 \times 2$ frequency tables, graphs, and figures, such

Prognostic factors predicting an unprovoked seizure recurrence in children and adults following a first unprovoked seizure (Protocol) 
as Kaplan-Meier curves, using indirect estimation measures, as described by Parmar 1998 and Tierney 2007).

\section{Assessment of heterogeneity}

We anticipate that clinical and statistical heterogeneity will be present between studies, due to the wide inclusion criteria for study design and participant populations. Consequently, we will use a random-effects model for the meta-analysis.

We will consider the clinical heterogeneity of included studies based on the study design, study duration, potential biases of the study, the participant population, the definition and measurement of the prognostic factor used (including any cutoff points), and the outcome measurement.

We will synthesise associations within clinically relevant subgroups (for example we will synthesise studies of a prospective and retrospective design separately). To assess statistical heterogeneity across studies included in each syntheses, we will inspect forest plots and quantify heterogeneity statistically using the $\mathrm{I}^{2}$ statistic and $\operatorname{Tau}^{2}$ (the estimate of between-study variance; (Snell 2016)).

\section{Assessment of reporting deficiencies}

We plan to examine publication bias for each meta-analysis, provided there were 10 or more studies, by visually examining asymmetry on funnel plots, and testing for asymmetry at the $10 \%$ level, using Egger's test for HRs, and Peters' test for ORs (Debray 2018; Sterne 2011).

\section{Data synthesis}

\section{Data synthesis and meta-analysis approaches}

We anticipate that relevant data for this review will be presented in a range of formats and levels of detail. Therefore, wherever possible, we aim to transform data to a common format for synthesis (see the 'Measures of association or predictive performance measures to be extracted' section), and we will examine the impact of any assumptions made when transforming data in a sensitivity analysis (e.g. if data are converted from one effect measure to another, or estimated from graphical figures).

Ideally, we would like to combine adjusted effect sizes from multivariable statistical models, to examine the association of each prognostic factor with the outcome, where study designs, participant populations, and prognostic factor definitions and cutoffs are sufficiently homogenous to synthesise (Debray 2017).

Realistically, we anticipate that the relationship between the prognostic factors and the outcome will be presented in the format of dichotomous data (univariate and unadjusted). If this is the case, we will calculate effect sizes based on the dichotomous data for the univariate relationship between each prognostic factor and the outcome, and pool these effect sizes where study designs, participant populations, and prognostic factor definitions and cutoffs are sufficiently homogenous to synthesise.

We will conduct meta-analyses using Review Manager 2014, with a random-effects, generic inverse variance meta-analysis model, which accounts for any between-study heterogeneity in the prognostic effect. We will summarise the meta-analysis by the pooled estimate (the average prognostic factor effect), its $95 \% \mathrm{Cl}$, the estimates of $\mathrm{I}^{2}$ and $\mathrm{Tau}^{2}$ (heterogeneity), and a $95 \%$ prediction interval for the prognostic effect in a single population (Riley 2011), which we will calculate in STATA version 15 (Stata).

If it is not appropriate to combine results using a meta-analysis (due to excess clinical heterogeneity or lack of appropriate data presented), we will present the results qualitatively, considering the strength and consistency of results, using the following schema:

- strong evidence of effect: consistent findings (defined as greater than $75 \%$ of studies showing the same direction of effect) in multiple studies at low risk of bias;

- moderate evidence of effect: consistent findings in multiple studies at high risk of bias, or one study at low risk of bias;

- limited evidence of effect: one study available;

- conflicting evidence of effect: inconsistent findings across studies;

- no effect: no association between participant expectations and the outcome of interest.

\section{Subgroup analysis and investigation of heterogeneity}

If appropriate and sufficient data are available, we will conduct separate meta-analyses based on distinct subgroups, such as prospectively and retrospectively designed studies, studies including adults and children (age group as defined in the individual study), and studies considering different seizure types. With regard to age, it is anticipated that we will present overall prognosis summary data separately, given that epidemiological and prognosis studies in epilepsy tend to study children and adults separately, with different overall prognosis and prognostic factors.

We will interpret results of subgroup meta-analysis, depending on how many studies contribute data to subgroups.

\section{Sensitivity analysis}

We will conduct sensitivity analyses in which, a. we include only studies that are judged, overall, to be at low risk of bias (Hayden 2013), and b. we examine the impact of any assumptions we make when transforming data.

We will also consider subgroup or sensitivity analyses to explore the impact of types of measurement approaches for assessing prognostic factors, or other methodological differences or shortcomings of the included studies.

\section{Conclusions and summary of findings}

We will use an approach modified from the GRADE framework to assess the overall certainty of evidence regarding the association of each prognostic factor with each outcome (Guyatt 2011; Hayden 2014; Huguet 2013; lorio 2015).

We will rate the overall strength of evidence as high, moderate, low, or very low considering the phase of the prognostic study and internal validity, size and precision of effect, heterogeneity, generalisability, and potential reporting bias.

\section{ACKNOWLEDGEMENTS}

We would like to acknowledge the Cochrane Epilepsy Group, the Cochrane Prognosis Methods Group, the Cochrane Mental Health and Neuroscience Network (Nuala Livingstone) and the Cochrane 
Editorial and Methods Department (Sarah Hodgkinson) for all their advice and support.
We, and the Cochrane Epilepsy Group, are grateful to the external peer reviewers for their time and comments. 


\section{RE F E R E N C E S}

\section{Additional references}

\section{Berg 2010}

Berg AT, Berkovic SF, Brodie MJ, Buchhalter J, Cross JH, van Emde Boas W, et al. Revised terminology and concepts for organization of seizures and epilepsies: report of the ILAE Commission on Classification and Terminology, 2005-2009. Epilepsia 2010;51(4):676-85. [DOI: 10.1111/ j.1528-1167.2010.02522.x] [PMID: 20196795]

\section{Debray 2017}

Debray TP, Damen JA, Snell KI, Ensor J, Hooft L, Reitsma JB, et al. A guide to systematic review and meta-analysis of prediction model performance. BMJ 2017;356:i6460. [DOI: 10.1136/ bmj.i6460] [PMID: 28057641]

\section{Debray 2018}

Debray TPA, Moons KG, Riley RD. Detecting small-study effects and funnel plot asymmetry in meta-analysis of survival data: a comparison of new and existing tests. Research Synthesis Methods 2018;9(1):41-50. [DOI: 10.1002/jrsm.1266] [PMID: 28975717]

\section{Geersing 2012}

Geersing GJ, Bouwmeester W, Zuithoff P, Spijker R, Leeflang M, Moons KG. Search filters for finding prognostic and diagnostic prediction studies in MEDLINE to enhance systematic reviews. PloS One 2012;7(2):e32844. [DOI: 10.1371/ journal.pone.0032844] [PMID: 22393453]

\section{Guyatt 2011}

Guyatt GH, Oxman AD, Schünemann HJ, Tugwell P, Knottnerus A. GRADE guidelines: a new series of articles in the Journal of Clinical Epidemiology. Journal of Clinical Epidemiology 2011;64(4):380-2. [DOI: 10.1016/ j.jclinepi.2010.09.011] [PMID: 21185693]

\section{Hauser 1993}

Hauser WA, Annegers JF, Kurland LT. Incidence of epilepsy and unprovoked seizures in Rochester, Minnesota: 1935-1984. Epilepsia 1993;34(3):453-68. [PMID: 8504780]

\section{Hayden 2013}

Hayden JA, van der Windt DA, Cartwright JL, Côté P, Bombardier C. Assessing bias in studies of prognostic factors. Annals of Internal Medicine 2013;158(4):280-6. [DOI: 10.7326/0003-4819-158-4-201302190-00009] [PMID: 23420236]

\section{Hayden 2014}

Hayden JA, Tougas ME, Riley R, Iles R, Pincus T. Individual recovery expectations and prognosis of outcomes in nonspecific low back pain: prognostic factor exemplar review. Cochrane Database of Systematic Reviews 2014, Issue 9. Art. No: CD011284. [DOI: 10.1002/14651858.CD011284]

\section{Huguet 2013}

Huguet A, Hayden JA, Stinson J, McGrath PJ, Chambers CT, Tougas ME, et al. Judging the quality of evidence in reviews of prognostic factor research: adapting the GRADE framework.
Systematic Reviews 2013;2:71. [DOI: 10.1186/2046-4053-2-71] [PMID: 24007720]

\section{Iorio 2015}

Iorio A, Spencer FA, Falavigna M, Alba C, Lang E, Burnand B, et al. Use of GRADE for assessment of evidence about prognosis: rating confidence in estimates of event rates in broad categories of patients. BMJ 2015;350:h870. [DOI: 10.1136/bmj.h870] [PMID: 25775931]

\section{Kwan 2010}

Kwan P, Arzimanoglou A, Berg AT, Brodie MJ, Hauser WA, Mathern G, et al. Definition of drug resistant epilepsy: consensus proposal by the ad hoc Task Force of the ILAE Commission on Therapeutic Strategies. Epilepsia 2010;51(6):1069-77. [DOI: 10.1111/j.1528-1167.2009.02397.x] [PMID: 19889013]

\section{Loiseau 1999}

Loiseau J, Picot MC, Loiseau P. Short-term mortality after a first epileptic seizure: a population-based study. Epilepsia 1999;40(10):1388-92. [DOI: 10.1111/j.1528-1157.1999.tb02010.x.] [PMID: 10528934]

\section{Marson 2005}

Marson A, Jacoby A, Johnson A, Kim L, Gamble C, Chadwick D, Medical Research Council MESS Study Group. Immediate versus deferred antiepileptic drug treatment for early epilepsy and single seizures: a randomised controlled trial. Lancet 2005;365(9476):2007-13. [DOI: 10.1016/S0140-6736(05)66694-9] [PMID: 15950714]

\section{Moher 2009}

Moher D, Liberati A, Tetzlaff J, Altman DG, PRISMA Group. Preferred reporting items for systematic reviews and metaanalyses: the PRISMA statement. BMJ 2009;339:b2535. [DOI: 10.1136/bmj.b2535] [PMID: 19622551]

\section{Moons 2014}

Moons KG, de Groot JA, Bouwmeester W, Vergouwe Y, Mallett S, Altman DG, et al. Critical appraisal and data extraction for systematic reviews of prediction modelling studies: the CHARMS checklist. PLoS Medicine 2014;11(10):e1001744. [DOI: 10.1371/journal.pmed.1001744] [PMID: 25314315]

\section{Neligan 2012}

Neligan A, Hauser WA, Sander JW. The epidemiology of the epilepsies. In: Stefan H, Theodore WH, editors(s). Epilepsy Part I (Handbook of Clinical Neurology vol. 107). Amsterdam: Elsevier, 2012:113-33. [DOI: 10.1016/B978-0-444-52898-8.00006-9] [PMID: 22938966]

\section{Neligan 2021}

Neligan A, Sander L, Nevitt SJ, Marson AG, Adan G, Pullen A. Prognosis of adults and children following a first unprovoked seizure. Cochrane Database of Systematic Reviews 2021.

\section{Ngugi 2011}

Ngugi AK, Kariuki SM, Bottomley C, Kleinschmidt I, Sander JW, Newton CR. Incidence of epilepsy: a systematic review and 
meta-analysis. Neurology 2011;77(10):1005-12. [DOI: 10.1212/

WNL.0b013e31822cfc90] [PMID: 21893672]

\section{Parmar 1998}

Parmar MK, Torri V, Stewart L. Extracting summary statistics to perform meta-analyses of the published literature for survival endpoints. Statistics in Medicine 1998;17(24):2815-34. [PMID: 9921604]

\section{Review Manager 2014 [Computer program]}

Nordic Cochrane Centre, The Cochrane Collaboration Review Manager 5 (RevMan 5). Version 5.3. Copenhagen: Nordic Cochrane Centre, The Cochrane Collaboration, 2014.

\section{Riley 2011}

Riley RD, Higgins JP, Deeks JJ. Interpretation of random effects meta-analyses. BMJ 2011;342:d549. [DOI: 10.1136/bmj.d549] [PMID: 21310794]

\section{Scheffer 2017}

Scheffer IE, Berkovic S, Capovilla G, Connolly MB, French J, Guilhoto L, et al. ILAE classification of the epilepsies: position paper of the ILAE Commission for Classification and Terminology. Epilepsia 2017;58(4):512-21. [DOI: 10.1111/ epi.13709] [PMID: 28276062]

\section{Snell 2016}

Snell KI, Hua H, Debray TP, Ensor J, Look MP, Moons KG, et al. Multivariate meta-analysis of individual participant data helped externally validate the performance and implementation of a prediction model. Journal of Clinical Epidemiology
2016;69:40-50. [DOI: 10.1016/j.jclinepi.2015.05.009] [PMID: 26142114]

\section{Stata [Computer program]}

Stata. Version 15. College Station, TX, USA: StataCorp, 2017. Available from www.stata.com

\section{Sterne 2011}

Sterne JA, Egger M, Moher D. Chapter 10: Addressing reporting biases. In: Higgins JP, Green S, editor(s), Cochrane Handbook for Systematic Reviews of Interventions version 5.1.0 (updated March 2011). The Cochrane Collaboration, 2011. Available from handbook-5-1.cochrane.org/.

\section{Symons 2002}

Symons MJ, Moore DT. Hazard rate ratio and prospective epidemiological studies. Journal of Clinical Epidemiology 2002;55(9):893-9. [DOI: 10.1016/s0895-4356(02)00443-2] [PMID: $12393077]$

\section{Tierney 2007}

Tierney JF, Stewart LA, Ghersi D, Burdett S, Sydes MR. Practical methods for incorporating summary time-to-event data into meta-analysis. Trials 2007;8:16. [DOI: 10.1186/1745-6215-8-16] [PMID: 17555582]

\section{West 2019}

West S, Nevitt SJ, Cotton J, Gandhi S, Weston J, Sudan A, et al. Surgery for epilepsy. Cochrane Database of Systematic Reviews 2019, Issue 6. Art. No: CD010541. [DOI: 10.1002/14651858.CD010541.pub3]

\section{APPENDICES}

\section{Appendix 1. Preliminary study selection, data extraction, and 'Risk of bias' forms}

We will use a modified version of the quality assessment strategy recommended by bias to assess the quality of included studies (Hayden 2013). This assessment will cover six domains of potential bias: study participation, study attrition, prognostic factors measurement (as detailed above), outcome measurement (seizure recurrence, death), study confounding, statistical analysis, and reporting. Our approach will assess the risk of bias by considering responses to the prompting items for all reported prognostic factors together (in addition to any missing or unclear information).

The issues to consider for judging the overall rating of risk of bias for each domain are listed below. We will provide study methods and comments, in addition to a rating of reporting within the review.

\section{Bias: study participation}

Goal: To judge the risk of selection bias (likelihood that the relationship between prognostic factors (PF) and outcome is different for participants and eligible non-participants)

\section{Issues to consider for judging overall rating of risk of bias}

Source of target population

The source population, or population of interest, is adequately described, including who the target population is (e.g. all people with a single unprovoked seizure, or people with a specific type of seizure, focal onset or generalised, or a single seizure occurring after a specific aetiology e.g. posttraumatic seizure), when (time period of study), where (tertiary care epilepsy clinic, First Seizure Clinic, general neurology or paediatric clinic, Accident and Emergency, primary care, community), and how (description of recruitment strategy - referrals from Accident and Emergency, primary care). 
Comprehensive description would include demographic (age, sex, date of seizure), relevant comorbidities and history (history of childhood febrile seizures, previous head injury, previous cerebrovascular accident, dementia), seizure type (focal, generalised, undefined), and whether any treatment (anti-epileptic medication) was initiated, and for how long.

Method used to identify population
Recruitment methodology is adequately described (direct referrals from primary care, Accident and Emergency), or is identified directly from the community (method of case ascertainment is clearly described).
Recruitment period Place of recruitment (setting - e.g. First Seizure Clinic, and geographic location) are adequately described.
Inclusion and exclusion criteria
Inclusion and exclusion criteria are adequately described, and define a discrete group with a single unprovoked seizure. In particular, people with provoked (acute symptomatic) seizures are specifically excluded, as people referred with a single seizure and have had a recurrence by the time of initial review in clinic are excluded, or people are included as a seizure relapse, with an accurate timeframe established.

Adequate study participation

The baseline characteristics of the individuals enrolled are adequately described. This would include age, sex, date of seizure, seizure type, and any identified risk factors for epilepsy or comorbidities.

Summary study participation: The study sample represents the population of interest on key characteristics, sufficient to limit potential bias of the observed relationship between PF and outcome (low, moderate, or high risk of bias).

\section{Bias: study attrition}

Goal: To judge the risk of attrition bias (likelihood that relationship between PF and outcome are different for completing and noncompleting participants

\section{Issues to consider for judging overall risk of bias}

Proportion of baseline sample available for analysis
Response rate (i.e. proportion of people in a cohort on whom we have complete follow-up seizure recurrence/mortality data) is adequate.
Attempts to collect information on participants who dropped out
Attempts to collect information on participants who were lost to follow-up are adequately described.

Reasons and potential impact of subjects lost Potential individual reasons for loss to follow-up are provided.

to follow-up

Outcome and prognostic factor information on those lost to follow-up
Baseline demographic characteristics and potential risk factors for seizure recurrence are adequately described in those lost to follow-up.

\section{Summary study attrition:}

Loss to follow-up (from baseline sample to study population analysed) is not associated with key characteristics (i.e. the study data adequately represent the sample) sufficient to limit potential bias to the observed relationship between PF and outcome (low, moderate, high risk of bias).

\section{Bias: prognostic (PF measurement)}

Goal: To assess the risk of measurement bias of prognostic factors related to seizure recurrence 


\section{Issues to consider for judging overall risk of bias}

Defintion of the PF Potential PFs, such as specific electroencephalogram (EEG) findings and specific neuro-imaging findings, are clearly and consistently defined.

Valid and reliable measurement of PF
Method of documentation of seizure recurrence is consistent for all individuals, i.e. use of seizure diaries, confirmed eye-witness accounts with accurate dates, and accurate seizure classification to avoid misclassification bias. Clear details of electroencephalogram (EEG) or neuroimaging methods provided, and classification of seizure type made using appropriate methods (e.g. using International League Against Epilepsy (ILAE) classifications (e.g. Berg 2010 or earlier versions)).
Method and setting of PF mea- The method of establishing seizure recurrence (e.g. seizure diary, eye-witness account) is consissurement

Proportion of data on PF avail- Adequate proportion of the cohort has complete data on potential PF (adequate to be judged, able for analysis based on context of the study).

Method used for missing data If used, appropriate methods of imputation are used for missing individual PFs.

\section{Summary prognostic factor measurement:}

PFs are adequately measured in study participants to sufficiently limit potential bias (low, moderate, high risk of bias).

\section{Bias: outcome measurement}

Goal: To assess the risk of bias related to seizure outcome (differential measurement of seizure outcome related to the baseline level of PF

\section{Issues to consider for judging overall risk of bias}

Definition of the outcome

Valid and reliable measurement of outcome
A clear definition of what constitutes a seizure recurrence is provided, including clear documentation of the time period between the index seizure and seizure recurrence, as well as clear documentation of seizure semiology.

The method of establishing seizure recurrence (outcome measurement) used is adequately valid and reliable, to limit misclassification bias. In particular, that sufficient clinical details are available regarding all potential seizures after the index seizure, to avoid misclassification of other differentials (syncope, non-epileptic attacks, provoked (acute symptomatic) seizures).

Method and setting of outcome measurement

Summary outcome measurement: outcome is adequately measured in study participants to sufficiently limit potential bias (low, moderate, high risk of bias).

\section{Bias: study confounding}

Goal: To judge the risk of bias due to confounding - i.e. the effect of a PF is distorted by another factor related to the PF and the risk of seizure recurrence or mortality

\section{Issues to consider for judging overall risk of bias}


(Continued)

Important confounders measured
All important potential confounders related to the risk of seizure recurrence, such as significant sleep deprivation, anti-seizure medication (ASM) treatment initiated, and premature mortality following a single seizure (such as important medical comorbidities, like ischaemic heart disease and diabetes mellitus) are measured.
Definition of the confounding factor
Clear definition of important confounding factors measured are provided (e.g. what constitutes significant sleep deprivation in the context of seizure recurrence).
Valid and reliable measurement of confounders
Measurement of all important confounders is adequately valid and reliable (e.g. confirmed documentation in previous medical records, clear EEG parameters for classification for non-diagnostic features).

\section{Method and setting of con- founding measurements \\ The method and setting of confounding measurements and recording are the same for all study participants.}

Method used for missing con- Appropriate methods are used if imputation is used for missing confounding factor data. founding factor data

Appropriate accounting for confounding
Important potential confounders are accounted for in study design (i.e. matching for key variables - age, sex, seizure semiology).

Summary study confounding: important potential confounders are appropriately accounted for, limiting potential bias with respect to the relationship between PFs and the outcome (low, moderate, high risk of bias).

\section{Bias: statistical analysis and reporting}

Goal: to judge the risk of bias related to the statistical analysis and presentation of results

\section{Issues to consider for judging overall rating of bias}

Presentation of analytical There is sufficient presentation of data to assess the appropriateness of the analysis used.
strategy

Model developmental strategy The strategy for prognostic model building is appropriate, and the statistical model used is appropriate for the study design.

Reporting of results

There is no manifest selective reporting of results.

Summary statistical analysis and reporting: the statistical analysis is appropriate for the design of the study, limiting potential for presentation of invalid or spurious results, and selective reporting is unlikely (low, moderate, high risk of bias).

\section{Appendix 2. CRS Web search strategy}

1. ((first or single or initial) ADJ4 seizure $\left.{ }^{\star}\right): A B, K W, K Y, M C, M H, T I$ AND CENTRAL:TARGET

2. (unprovoked or untreated):AB,KW,KY,MC,MH,TI AND CENTRAL:TARGET

3. \#1 AND \#2

4. ((first or single or unprovoked) adj3 seizure $\left.{ }^{\star}\right)$ :TI AND CENTRAL:TARGET

5. \#3 OR \#4

6. MESH DESCRIPTOR Diagnosis EXPLODE ALL AND CENTRAL:TARGET

Prognostic factors predicting an unprovoked seizure recurrence in children and adults following a first unprovoked seizure (Protocol) 
7. MESH DESCRIPTOR Risk Factors EXPLODE ALL AND CENTRAL:TARGET

8. MESH DESCRIPTOR Recurrence EXPLODE ALL AND CENTRAL:TARGET

9. MESH DESCRIPTOR Mortality EXPLODE ALL AND CENTRAL:TARGET

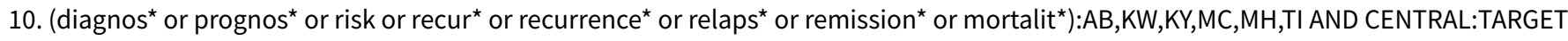

11. \#6 OR \#7 OR \#8 OR \#9 OR \#10

12. MESH DESCRIPTOR Epilepsy EXPLODE ALL AND CENTRAL:TARGET

13. (epilep $\left.{ }^{\star}\right): A B, K W, K Y, M C, M H, T I$ AND CENTRAL:TARGET

14. MESH DESCRIPTOR Seizures AND CENTRAL:TARGET

15. \#12 OR \#13 OR \#14

16. \#11 AND \#15

17. MESH DESCRIPTOR Epilepsy EXPLODE ALL WITH QUALIFIER DI AND CENTRAL:TARGET

18. MESH DESCRIPTOR Seizures WITH QUALIFIER DI AND CENTRAL:TARGET

19. \#16 OR \#17 OR \#18

20. (Validat* OR Rule*):AB,KW,KY,MC,MH,TI AND CENTRAL:TARGET

21. (Predict*):TI AND CENTRAL:TARGET

22. $\left(\right.$ Predict $^{\star}$ AND (Outcome ${ }^{\star}$ or Risk ${ }^{\star}$ or Model $\left.^{\star}\right)$ ):AB,KW,KY,MC,MH,TI AND CENTRAL:TARGET

23. ((History or Variable ${ }^{\star}$ or Criteria or Scor ${ }^{\star}$ or Characteristic ${ }^{\star}$ or Finding ${ }^{\star}$ or Factor ${ }^{\star}$ ) and (Predict* or Model $^{\star}$ or Decision $^{\star}$ or Identif ${ }^{\star}$ or Prognos $\left.{ }^{\star}\right)$ ):AB,KW,KY,MC,MH,TI AND CENTRAL:TARGET

24. (Decision $\left.{ }^{\star}\right): A B, K W, K Y, M C, M H, T I$ AND CENTRAL:TARGET

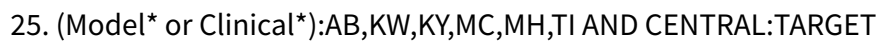

26. MESH DESCRIPTOR Logistic Models AND CENTRAL:TARGET

27. \#25 OR \#26

28. \#24 AND \#27

29. (Prognostic and (History or Variable* or Criteria or Scor ${ }^{\star}$ or Characteristic ${ }^{\star}$ or Finding* or Factor ${ }^{\star}$ or Model $\left.{ }^{\star}\right)$ ):AB, KW, KY,MC,MH,TI AND CENTRAL:TARGET

30. \#20 OR \#21 OR \#22 OR \#23 OR \#28 OR \#29

31. (Predict* OR Scor^ OR Observ*):TI,AB AND CENTRAL:TARGET

32. MESH DESCRIPTOR Predictive Value of Tests AND CENTRAL:TARGET

33. MESH DESCRIPTOR Observer Variation AND CENTRAL:TARGET

34. \#31 OR \#32 OR \#33

35. (Stratification OR Discrimination OR Discriminate OR "c-statistic" OR "c statistic" OR "Area under the curve" OR AUC OR Calibration OR Indices OR Algorithm OR Multivariable):AB,KW,KY,MC,MH,TI AND CENTRAL:TARGET

36. MESH DESCRIPTOR ROC Curve AND CENTRAL:TARGET

37. \#35 OR \#36

38. \#30 OR \#34 OR \#37

39. \#19 OR \#38

Prognostic factors predicting an unprovoked seizure recurrence in children and adults following a first unprovoked seizure (Protocol) 
40. \#5 AND \#39

41. (cancer ${ }^{\star}$ or glioma* or glioblast $^{\star}$ or neoplasm ${ }^{\star}$ or tumor $^{\star}$ or tumour ${ }^{\star}$ or stroke):TI AND CENTRAL:TARGET

42. ((eclamp* or alcohol withdraw* or febril*) NOT "non-febril $\left.{ }^{\star} "\right):$ TI AND CENTRAL:TARGET

43. \#41 OR \#42

44. \#40 NOT \#43

\section{Appendix 3. MEDLINE Ovid (from 1946)}

This includes the search filters recommended by the Cochrane Prognosis Methods Group (Geersing 2012):

1. ((first or single or initial) adj4 seizure?).tw.

2. (unprovoked or untreated).tw.

3. 1 and 2

4. ((first or single or unprovoked) adj3 seizure?).ti.

5. 3 or 4

6. exp Diagnosis/ or exp risk factors/ or exp RECURRENCE/ or exp Mortality/

7. (diagnos $\$$ or prognos $\$$ or risk or recur? or recurrence? or relaps\$ or remission\$ or mortalit\$).tw.

8. 6 or 7

9. exp Epilepsy/ or epilep*.tw. or seizures/ [seizures deliberately not exploded]

10. 8 and 9

11. exp Epilepsy/di or seizures/di [seizures deliberately not exploded]

12. 10 or 11

13. Validat\$.mp. or Predict\$.ti. or Rule\$.mp. or (Predict\$ and (Outcome\$ or Risk\$ or Model\$)).mp. or ((History or Variable\$ or Criteria or Scor\$ or Characteristic\$ or Finding\$ or Factor\$) and (Predict\$ or Model\$ or Decision\$ or Identif\$ or Prognos\$)).mp. or (Decision\$.mp. and ((Model\$ or Clinical\$).mp. or Logistic Models/)) or (Prognostic and (History or Variable\$ or Criteria or Scor\$ or Characteristic\$ or Finding\$ or Factor\$ or Model\$)).mp. [mp=title, abstract, original title, name of substance word, subject heading word, floating sub-heading word, keyword heading word, organism supplementary concept word, protocol supplementary concept word, rare disease supplementary concept word, unique identifier, synonyms]

14. Predict\$.ti,ab. or Predictive value of tests/ or Scor\$.ti,ab. or Observ\$.ti,ab. or observer variation/

15. "Stratification".mp. or roc curve/ or "Discrimination".mp. or "Discriminate".mp. or "c-statistic".mp. or "c statistic".mp. or "Area under the curve".mp. or "AUC".mp. or "Calibration".mp. or "Indices".mp. or "Algorithm".mp. or "Multivariable".mp. [mp=title, abstract, original title, name of substance word, subject heading word, floating sub-heading word, keyword heading word, organism supplementary concept word, protocol supplementary concept word, rare disease supplementary concept word, unique identifier, synonyms]

16. 13 or 14 or 15

17. 12 or 16

18. 5 and 17

19. $\exp { }^{\star}$ Neoplasms/ or exp *Stroke/

20. (cancer\$ or glioma $\$$ or glioblast $\$$ or neoplasm $\$$ or tumor $\$$ or tumour\$ or stroke).ti.

21. exp *Pre-Eclampsia/ or exp *Eclampsia/

22. exp *alcohol withdrawal seizures/ or exp *seizures, febrile/

23. ((eclamp\$ or alcohol withdraw\$ or febril\$) not non-febril\$).ti.

Prognostic factors predicting an unprovoked seizure recurrence in children and adults following a first unprovoked seizure (Protocol) 
24. or/19-23

25. 18 not 24

26. exp animals/ not humans.sh.

27.25 not 26

28. 27 not case reports.pt.

29. remove duplicates from 28

\section{Appendix 4. SCOPUS search strategies}

\section{Subject search}

((()((TITLE-ABS-KEY((first OR single OR initial) PRE/4 seizure) AND TITLE-ABS-KEY(unprovoked OR untreated)) OR (TITLE((first OR single OR unprovoked) PRE/3 seizure))) AND (((TITLE-ABS-KEY(diagnos* OR prognos* OR risk OR recur OR recurrence OR relaps ${ }^{\star}$ OR remission OR mortalit*)) AND ((TITLE-ABS-KEY(epilep* OR "infantile spasm" OR "ring chromosome 20" OR "R20" OR "myoclonic encephalopathy" OR "pyridoxine dependency") OR (TITLE-ABS-KEY(syndrome) W/2 (aicardi OR angelman OR doose OR dravet OR janz OR jeavons OR "landau kleffner" OR "lennox gastaut" OR ohtahara OR panayiotopoulos OR rasmussen OR rett OR "sturge weber" OR tassinari OR "unverricht lundborg" OR west)) OR TITLE(seizure OR convuls*) OR (TITLE-ABS-KEY(lafora*) W/4 (disease OR epilep*) AND NOT (TITLE(dog OR canine) OR INDEXTERMS(dog OR canine)))) AND NOT (TITLE( ${ }^{*}$ eclampsia) OR INDEXTERMS(*eclampsia)))) OR ((TITLE-ABS-KEY(Validat* OR Rule*) OR TITLE(Predict*)) OR (TITLE-ABS-KEY(Predict* AND (Outcome* OR Risk* OR Model*))) OR ((TITLE-ABS-KEY(History OR Variable* OR Criteria OR Scor ${ }^{\star}$ OR Characteristic ${ }^{\star}$ OR Finding ${ }^{\star}$ OR Factor $\left.\left.{ }^{\star}\right)\right)$ AND (TITLE-ABS-KEY(Predict ${ }^{\star}$ OR Model ${ }^{\star}$ OR Decision ${ }^{\star}$ OR Identif $^{\star}$ OR Prognos $\left.\left.{ }^{\star}\right)\right)$ ) OR (TITLE-ABS-KEY(Decision* AND (Model* OR Clinical* OR "Logistic Model*"))) OR (TITLE-ABS-KEY(Prognostic AND (History OR Variable* OR Criteria OR Scor ${ }^{\star}$ OR Characteristic ${ }^{\star}$ OR Finding* OR Factor ${ }^{\star}$ OR Model $\left.{ }^{\star}\right)$ )) OR (TITLE-ABS(Predict* OR Scor ${ }^{\star}$ OR Observ ${ }^{\star}$ ) OR TITLEABS-KEY("Predictive value of tests" OR "observer variation")) OR (TITLE-ABS-KEY(Stratification OR "roc curve" OR Discrimination OR Discriminate OR "c-statistic" OR "c statistic" OR "Area under the curve" OR AUC OR Calibration OR Indices OR Algorithm OR Multivariable))))) AND NOT (TITLE(animal* OR mouse OR mice OR rat OR dog OR canine) AND NOT TITLE(human* OR patient OR child* OR infant* OR adolescen* OR adult OR elderly OR man OR men OR male OR wom?n OR female))) AND ((TITLE-ABS((randomiz* OR randomis* OR controlled OR placebo OR blind* OR unblind* OR "parallel group" OR crossover OR "cross over" OR cluster OR "head to head") W/4 (analy* OR investigat* OR method OR procedure OR study OR studies OR trial))) OR ((( TITLE-ABS(("before and after" OR cohort OR comparative OR "cross section*" OR "follow up" OR longitudinal OR multicenter OR observation* OR prospective OR quasicontrol* OR "quasi control*" OR quasiexperiment* or "quasi experiment ${ }^{\star}$ " OR quasirandom* OR "quasi random*" OR "record linkage" OR retrospective OR "time series") W/4 (analy ${ }^{\star}$ OR investigat ${ }^{\star}$ OR method OR procedure OR study OR studies OR trial))) OR (TITLE-ABS(case* W/3 (comparison ${ }^{\star}$ OR control ${ }^{\star}$ OR series))) OR (TITLE-ABS((clinical OR epidemiologic OR evaluation OR validation) PRE/3 (study OR studies OR trial))) OR (ABS("time points" W/3 (over OR multiple OR three OR four OR five OR six OR seven OR eight OR nine OR ten OR eleven OR twelve OR month OR hour OR day OR "more than"))) OR (ABS(control W/3 (area OR cohort OR compare* OR condition OR design OR group OR intervention OR participant OR study))) OR (TITLE-ABS("control year" OR "experimental year" OR "control period" OR "experimental period")) OR (TITLE-ABS((strategy OR strategies) W/2 (improv* OR education*)))) OR (TITLE-ABS-KEY((single OR doubl* OR tripl* OR treb*) PRE/3 (blind* OR mask* ))) OR (TITLEABS-KEY("4 arm" OR "four arm"))))) AND NOT (TITLE(case PRE/0 (report OR study OR studies)))) AND NOT (TITLE(cancer* OR glioma* OR glioblast* OR neoplasm* OR tumor* OR tumour* OR stroke OR eclamp* OR "alcohol withdraw*" OR febril*) AND NOT TITLE("non-febril*"))

\section{Citation search}

\section{Documents that cite}

PMID(26780937 OR 18184149 OR 2864487 OR 1978114 OR 26215392 OR 26222507 OR 24055222 OR 10528934 OR 23181965 OR 25676481 OR 24691297 OR 8692621 OR 27680779)

LIMIT-TO(DOCTYPE, "ar" ) OR LIMIT-TO(DOCTYPE, "cp") AND (EXCLUDE(EXACTKEYWORD, "Animals") OR EXCLUDE(EXACTKEYWORD, "Nonhuman") OR EXCLUDE(EXACTKEYWORD, "Case Report"))

[DOCTYPE, "ar" = Article, DOCTYPE, "cp" = Conference paper]

\section{Cited documents}

Bell GS, Neligan A, Giavasi C, Keezer MR, Novy J, Peacock JL, et al. Outcome of seizures in the general population after 25 years: a prospective follow-up, observational cohort study. Journal of Neurology, Neurosurgery and Psychiatry 2016; 87(8): 843-50. PubMed ID: 26780937. DOI: 10.1136/jnnp-2015-312314.

Berg AT. Risk of recurrence after a first unprovoked seizure. Epilepsia 2008; 49(Suppl. 1): 13-8. PubMed ID: 18184149. DOI: 10.1111/ j.1528-1167.2008.01489.x.

Elwes RDC, Chesterman P, Reynolds EH. Prognosis after a first untreated tonic-clonic seizure. Lancet 1985; 326(8458): 752-3. PubMed ID: 2864487. DOI: 10.1016/S0140-6736(85)90631-2. 
Hart YM, Sander JWAS, Shorvon SD, Johnson AL. National General Practice Study of Epilepsy: recurrence after a first seizure. Lancet 1990; 336(8726): 1271-4. PubMed ID: 1978114. DOI: 10.1016/0140-6736(90)92960-P.

Kim H, Oh A, De Grauw X, De Grauw TJ. Seizure recurrence in developmentally and neurologically normal children with a newly diagnosed unprovoked seizure. Journal of Child Neurology 2016; 31(4): 421-5. PubMed ID: 26215392. DOI: 10.1177/0883073815596616.

Lawn N, Chan J, Lee J, Dunne J. Is the first seizure epilepsy - and when? Epilepsia 2015; 56(9): 1425-31. PubMed ID: 26222507. DOI: 10.1111/ epi.13093.

Lawn N, Kelly A, Dunne J, Lee J, Wesseldine A. First seizure in the older patient: clinical features and prognosis. Epilepsy Research 2013; 107(1-2): 109-14. PubMed ID: 24055222. DOI: 10.1016/j.eplepsyres.2013.08.009.

Loiseau J, Picot MC, Loiseau P. Short-term mortality after a first epileptic seizure: a population-based study. Epilepsia 1999; 40(10): 1388-92. PubMed ID: 10528934. DOI: 10.1111/j.1528-1157.1999.tb02010.x.

Maillard L, Jonas J, Boyer R, Frismand S, Mathey G, Vignal JP, et al. One-year outcome after a first clinically possible epileptic seizure: predictive value of clinical classification and early EEG. Neurophysiologie Clinique 2012; 42(6): 355-62. PubMed ID: 23181965. DOI: 10.1016/ j.neucli.2012.07.002.

Mizorogi S, Kanemura H, Sano F, Sugita K, Aihara M. Risk factors for seizure recurrence in children after first unprovoked seizure. Pediatrics International 2015; 57(4): 665-9. PubMed ID: 25676481. DOI: 10.1111/ped.12600.

Pereira C, Resende C, Fineza I, Robalo C. A 15-year follow-up of first unprovoked seizures: a prospective study of 200 children. Epileptic Disorders 2014; 16(1): 50-5. PubMed ID: 24691297. DOI: 10.1684/epd.2014.0643.

Shinnar S, Berg AT, Moshe SL, O'Dell C, Alemany M, Newstein D, et al. The risk of seizure recurrence after a first unprovoked afebrile seizure in childhood: An extended follow-up. Pediatrics 1996; 98(2): 216-25. PubMed ID: 8692621.

Zhang L, Huang Z, Tang J, Li Y. Risk factors following first spontaneous epileptic seizure in children below 3 years of age. International Journal of Neuroscience 2017; 127(9): 745-51. PubMed ID: 27680779. DOI: 10.1080/00207454.2016.1243105.

\section{Appendix 5. ClinicalTrials.gov search strategy}

diagnosis OR prognosis OR risk OR recurrence OR relapse OR remission OR mortality | (first OR single OR initial OR unprovoked OR untreated) AND (epilepsy OR epileptic OR seizure)

\section{Appendix 6. ICTRP search strategy}

epilepsy AND prognosis OR epilepsy AND prognostic OR epilepsy AND recurrence OR epilepsy AND relapse OR epilepsy AND remission OR epilepsy AND mortality

\section{H I S T O R Y}

Protocol first published: Issue 1, 2021

\section{CONTRIBUTIONS OF AUTHORS}

AN and GA developed the protocol with input from other named authors, AN and GA intend to carry out data extraction, quality assessment and data synthesis with the support of SJN and AGM.

\section{DECLARATIONSOF INTEREST}

GA: none known

AN: AN has received speaker honoria from Eisai Ltd and UCB Pharma.

SJN: none known

AP: none known

JWS: JWS's department has received grants from UCB Pharma. He has received honoraria from Zobenix, Arvelle and UCB for participating on an advisory board for drug development.

AGM: a consortium of pharmaceutical companies (GSK, EISAI, UCB Pharma) funded the National Audit of Seizure Management in Hospitals (NASH) through grants paid to the University of Liverpool. Professor Tony Marson is part funded by National Institute for Health Research Collaboration for Leadership in Applied Health Research and Care North West Coast (NIHR CLAHRC NWC). 


\section{SOURCES OF SUPPORT}

\section{Internal sources}

- No sources of support supplied

\section{External sources}

- National Institute for Health Research (NIHR), UK

This protocol was funded by the National Institute for Health Research (NIHR) [Clinically effective treatments for central nervous system disorders in the NHS, with a focus on Epilepsy and Movement Disorders (SRPG project 16/114/26)]. The views expressed are those of the author(s) and not necessarily those of the NIHR or the Department of Health and Social Care. 